\title{
Mini-filaments - small-scale analogues of solar eruptive events?
}

\author{
Carsten Denker ${ }^{1}$ and Alexandra Tritschler ${ }^{2}$ \\ ${ }^{1}$ Astrophysikalisches Institut Potsdam, An der Sternwarte 16, D-14482 Potsdam, Germany \\ email: cdenker@aip.de \\ ${ }^{2}$ National Solar Observatory/Sacramento Peak, Sunspot, NM 88349, USA \\ email: ali@nso.edu
}

\begin{abstract}
Mini-filaments are a small-scale phenomenon of the solar chromosphere, which frequently occur across the entire disk (see e.g. Wang, Li, Denker, et al. 2000). They share a variety of characteristics with their larger-scale cousins and may serve as a proxy for more complex systems. They play an important role in the energy and mass supply to the corona. In the case of small-scale eruptive filaments, only a single, small-scale loop system is involved. Furthermore, they are supported by simple magnetic field configurations (see Livi, Wang \& Martin 1985), either magnetic bipoles or well-defined multipoles, easing their theoretical description. Since mini-filaments are small (just a few tens of seconds of arc) but highly dynamic (eruptions can occur within just a few minutes), they are an ideal target for high-resolution two-dimensional spectroscopy. We present a preliminary analysis of two-dimensional $\mathrm{H} \alpha$ spectroscopic data accompanied by broad-band speckle-restored images to demonstrate that chromospheric smallscale phenomena can serve as building blocks for our understanding of solar eruptive events such as filament/prominence eruptions and even coronal mass ejections (CMEs).
\end{abstract}

Keywords. Sun: photosphere - Sun: chromosphere - Sun: filaments - Sun: magnetic fields

\section{Observations}

The observations were obtained with the Göttingen Fabry-Pérot Interferometer at the Vacuum Tower Telescope located at Observatorio del Teide, Tenerife. About 50 data sets were taken from 07:55 to 08:32 UT on 7 August 2008. The target was a small emerging magnetic dipole at heliographic coordinates $21.7^{\circ}$ East and $1.1^{\circ}$ South. The dipole was short-lived (less than a day) showing up as an inconspicuous brightening in $\mathrm{H} \alpha$ fulldisk images. The data consist of narrow-band $(3.5 \mathrm{pm})$ filtergrams equidistantly spaced $(2.21 \mathrm{pm})$ spanning the chromospheric absorption line $\mathrm{H} \alpha$ and simultaneous broad-band $(5 \mathrm{~nm})$ images at $630 \mathrm{~nm}$ for post-facto image restoration. The broad-band images are restored using the speckle masking method and the narrow-band filtergrams are improved using speckle deconvolution. For this reason, multiple frames have to be taken per wavelength position resulting in a total of $8 \times 61=488$ frames per scan. The data acquisition rate was about 15 frames/s resulting in a cadence of about $34 \mathrm{~s}$. The image scale was $0.11^{\prime \prime} /$ pixel corresponding to a field-of-view (FOV) of $77^{\prime \prime} \times 58^{\prime \prime}$.

\section{Results}

Speckle masking imaging is a powerful tool to recover spatial information down to the diffraction limit of solar telescopes. The speckle-restored broad-band image in Fig. 1d shows a quiet Sun area, which contains a small emerging magnetic bipole. Small-scale brightenings down to sizes of about $0.25^{\prime \prime}$ indicate the presence of individual flux tubes or small clusters thereof. Small-scale dark features, so called magnetic knots, point to more 


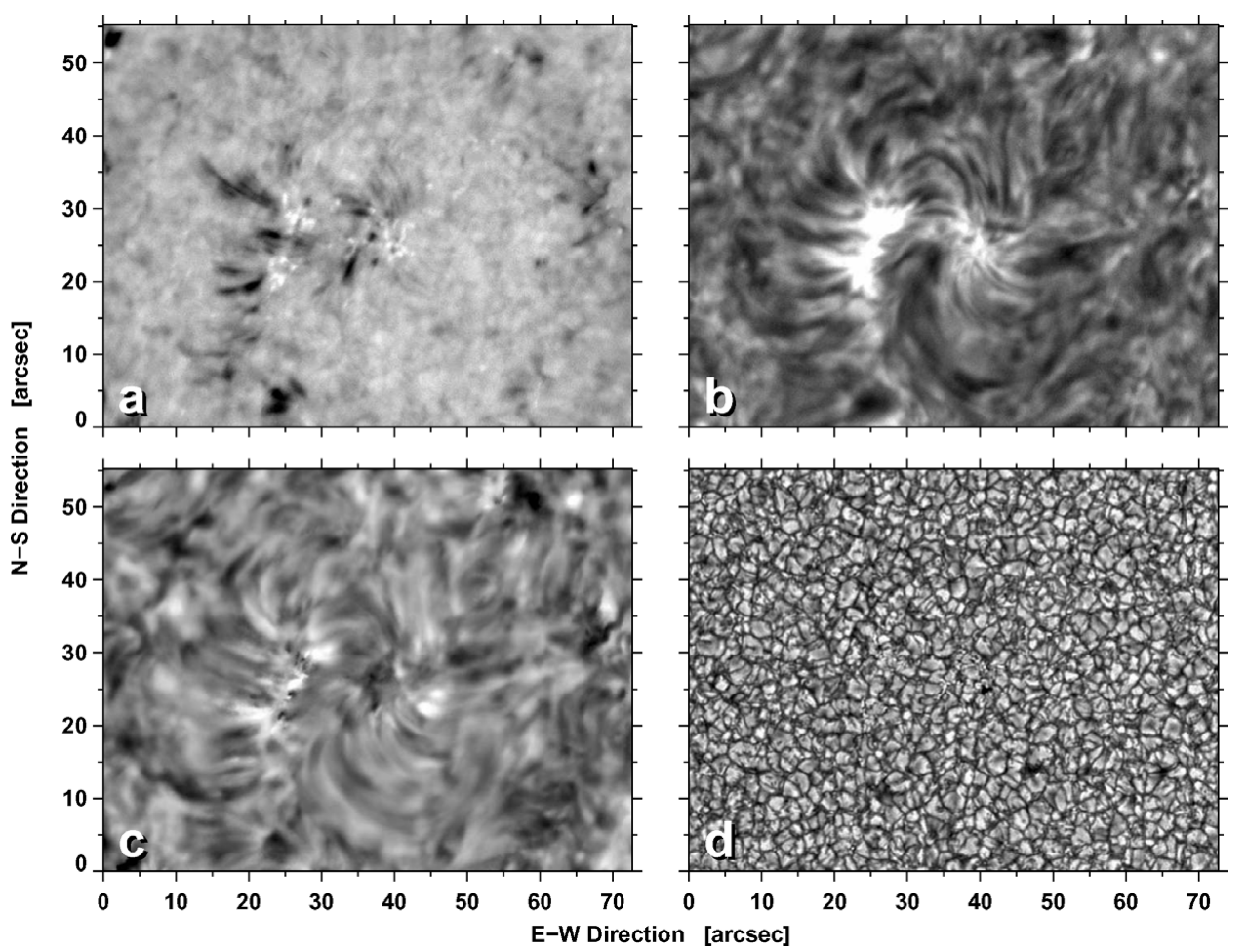

Figure 1. Emerging magnetic bipole observed on 7 August 2008. Narrow-band H $\alpha$ line wing (a) and line core (b) filtergram. (c) H $\alpha$ Doppler velocity map and (d) speckle-restored broad-band image showing small-scale magnetic features (filigree and magnetic knots).

concentrated magnetic fields. $\mathrm{H} \alpha$ line core filtergrams (Fig. 1b) show the mini-filament loop system connecting the emerging magnetic bipole. Small-scale brightenings, so called Ellerman bombs, are clearly visible in the far blue wing of the $\mathrm{H} \alpha$ line (Fig. 1a). These features are cospatial with small-scale brightenings in the speckle-restored broad-band image. The highest velocities exceeding $5 \mathrm{~km} / \mathrm{s}$ (downward flows) are encountered in the vicinity of the mini-filament's footpoints (see the $\mathrm{H} \alpha$ line core Doppler velocity map in Fig.1c). Upward flows occur along the neutral line dividing the $\mathrm{H} \alpha$ brightenings.

We presented a glimpse at preliminary results concerning small-scale emerging flux elements, which induce the formation of mini-filaments. Even though small-scale magnetic bipoles and multipoles are involved in the formation of mini-filaments, not all configurations are conducive to their formation. In our example the mini-filament did not form along the magnetic neutral line of the emerging dipole, it much more resembled arch filament systems observed in emerging flux regions. The footpoints of the mini-filament are rooted in the regions of opposite polarity and show brightenings in the line core $\mathrm{H} \alpha$. The observed mini-filaments in this magnetic configuration appeared to be stable, while the flux emergence lasted. We are currently analyzing further data sets to find magnetic configurations, which lead to more active mini-filaments and possibly to their eruption.

\section{References}

Livi, S. H. B., Wang, J., \& Martin, S. F. 1985, AuJPh 38, 855

Wang, J., Li, W., Denker, C., Lee, C., Wang, H., Goode, P. R., McAllister, A., \& Martin, S. F. 2000, ApJ 530, 1071 